\title{
Chloride Ion Corrosion Pattern and Mathematical Model for C60 High-Strength Concrete after Freeze-Thawing Cycles
}

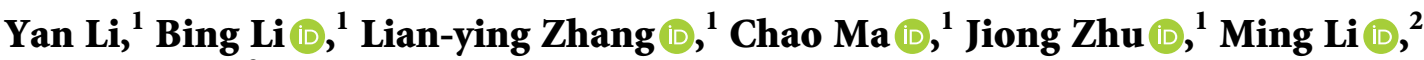 \\ and Hai Pu ${ }^{2}$ \\ ${ }^{1}$ School of Civil Engineering, Xuzhou Institute of Technology, Xuzhou 221008, Jiangsu, China \\ ${ }^{2}$ State Key Laboratory of Geomechanics and Deep Underground Engineering, China University of Mining \& Technology, \\ Xuzhou 221008, Jiangsu, China
}

Correspondence should be addressed to Bing Li; libing_libing_2007@163.com

Received 20 February 2021; Revised 22 March 2021; Accepted 27 March 2021; Published 8 April 2021

Academic Editor: Chunyang Zhang

Copyright (C) 2021 Yan Li et al. This is an open access article distributed under the Creative Commons Attribution License, which permits unrestricted use, distribution, and reproduction in any medium, provided the original work is properly cited.

In this study, the porosities of C60 high-strength concrete after 0,30, 60, and 90 freeze-thaw cycles determined via the water retention method are $1.30 \%, 3.65 \%, 5.14 \%$, and $7.34 \%$, respectively. Furthermore, a mathematical model of porosity varying with the number of freeze-thaw cycles is established. Using an artificial environment simulation experimental system and the natural diffusion method, the chloride diffusion law of C60 high-strength concrete after 0, 30, 60, and 90 freeze-thaw cycles is obtained. The corresponding diffusion coefficients are calculated based on the experimental results and Fick's law, where $0.3431 \times 10^{-12}$ $0.5288 \times 10^{-12}$, and $0.6712 \times 10^{-12}$, and $0.8930 \times 10^{-12} \mathrm{~m}^{2} / \mathrm{s}$ are obtained, respectively, and a mathematical model of diffusion coefficient with freeze-thawing is established. Transport control equations comprising solution flow and solute migration control equations are established for chloride ions in concrete after freeze-thawing cycles. The equations consider the effects of freezethawing, solution pressure, solution concentration, solution density, convection, mechanical dispersion, and chemisorption on chloride ion transport in concrete. Using COMSOL numerical software, the transport control equations for chloride ions are solved using a real concrete numerical model, and the chloride ion corrosion process in concrete after freeze-thaw cycles is simulated. The simulation results are consistent with the experimental values.

\section{Introduction}

With the continuous increase in energy demand, mineral resources extraction has gradually shifted to deeper strata, and the development depth of vertical shafts has increased consequently, along with the increase in the cross-sectional size of the shaft [1-12]. For the development of deep and thick unstable aquifer mines, the artificial ground freezing method is widely used [13-15]. When constructing using the freezing method, the freezing shaft lining is crucial, and its main function is to block groundwater and withstand the temporary load to ensure the safety and smooth operation of shaft sinking construction [16-19]. The structural forms of freezing shaft lining primarily include single-layer reinforced concrete, double-layer reinforced concrete, and reinforced concrete composite shaft lining. Currently, concrete materials are essential in the construction of all freezing shaft lining. With the increase in mining depth, high-strength and high-performance concrete materials such as C60 and C70 have been used for freezing shaft lining, and many scholars have conducted relevant studies [20-24].

As the freezing shaft lining is generally cast on site, pouring concrete will produce a large amount of heat of hydration, and the heat of hydration will contribute to part of the freezing lining temperature. After the heat of hydration dissipates, the freezing lining will refreeze, and the temperature will decrease; therefore, the freezing shaft lining will be in a freeze-thaw environment with a changing temperature field, posing a significant challenge to the strength and stability of the freezing lining. Hence, many scholars have investigated the effect of temperature on the rupture of vertical shaft lining [20-32]. Reinforced concrete structures for freezing shaft lining not only are affected by temperature and stress fields but also are susceptible to the 
corrosive effects of the subsurface environment. For example, sulfate has a certain corrosive and destructive effect on concrete materials, and the intrusion of chloride ions accelerates the rusting of reinforcing steel bars, thereby affecting the stability of the structure; this is closely associated with the durability problem of concrete structures and materials [33-37].

By understanding the corrosion law of chloride ions in C60 high-strength concrete after freeze-thawing, we aim to establish control equations for chloride ion transport in concrete considering freeze-thawing, solution pressure, solution concentration, solution density, convection, mechanical dispersion, and chemisorption and verify them by numerical calculations to provide some theoretical basis for the design, construction, and safe use evaluation of freezing shaft lining.

\section{Porosity of Concrete after Freeze- Thawing Cycles}

2.1. Materials and Mix Proportions. They are cement: 42.5 grade ordinary silicate cement supplied by Xuzhou Zhonglian Cement Plant, the composition of which is shown in Table 1; fine aggregate: river sand with a fineness modulus of 2.8 , good gradation, apparent density of $2679 \mathrm{~kg} / \mathrm{m}^{3}$, and silt content of 2.49\%; and coarse aggregate: stone, particle size of 5-20 mm, apparent density of $2719 \mathrm{~kg} / \mathrm{m}^{3}$, and silt content of $0.54 \%$. The mix proportions of concrete were $453 \mathrm{~kg} / \mathrm{m}^{3}$ cement, $740 \mathrm{~kg} / \mathrm{m}^{3}$ sand, $1112 \mathrm{~kg} / \mathrm{m}^{3}$ stone, and $145 \mathrm{~kg} / \mathrm{m}^{3}$ water; the water-cement ratio was 0.32 . The compressive strength of the sample was $57.0 \mathrm{MPa}$ after $28 \mathrm{~d}$ of standard curing.

2.2. Test Procedure. The test procedure, as shown in Figure 1, is as follows.

Three cylindrical concrete samples of diameter $d 100 \mathrm{~mm}$ and height $h 50 \mathrm{~mm}$ (standard curing for $28 \mathrm{~d}$ ) were used. Their actual height was measured, and then they were subjected to $0,30,60$, and 90 freeze-thaw cycles (based on the GBT50082-2009 standard, China, shown in Figure 1(a)) and porosity tests (shown in Figures $1(\mathrm{a})-1(\mathrm{~d})$ ).

The porosity test was performed using the water saturation method. The freeze-thaw treated samples were first dried at $40^{\circ} \mathrm{C}$ for $48 \mathrm{~h}$ (shown in Figure $1(\mathrm{~b})$ ) and then weighed using an electronic balance, placed in a water tank with a $16 \mathrm{~mm}$ diameter PVC pipe underneath, and filled with room-temperature water such that the water surface was $4-5 \mathrm{~mm}$ above the bottom of the sample (shown in Figure 1(c)). The samples were removed at $5,10,30,60,120,720$, and $1440 \mathrm{~min}$, respectively, and then weighed (shown in Figure $1(\mathrm{~d})$ ) and placed back immediately until all intervals were completed. Finally, the samples were completely submerged, and their water-saturated mass was measured after another $48 \mathrm{~h}$.

2.3. Porosity. Porosity $\phi$ is calculated using the following equation:

$$
\phi=\frac{V_{\mathrm{ws}}}{V_{\mathrm{cs}}},
$$

where $\phi$ is the porosity, $V_{\text {ws }}$ is the volume of water in the sample when saturated, and $V_{\mathrm{cs}}$ is the actual volume of the sample.

$V_{\text {ws }}$ and $V_{\mathrm{cs}}$ are calculated using the two following equations, respectively:

$$
\begin{aligned}
& V_{\mathrm{ws}}=\frac{m_{\mathrm{ws}}}{\rho_{\mathrm{w}}}, \\
& V_{\mathrm{cs}}=\frac{1}{4} \pi d^{2} h_{c} .
\end{aligned}
$$

In equation (2), $m_{\mathrm{ws}}$ is the mass of water in the sample when saturated, and $\rho_{\mathrm{w}}$ is the density of water; in equation (3), the diameter of the sample $d=100 \mathrm{~mm}$, and $h_{\mathrm{c}}$ is the actual height of the sample.

$m_{\mathrm{ws}}$ is calculated using the following equation:

$$
w=\frac{m_{1}-m_{0}}{m_{0}},
$$

where $m_{1}$ is the mass of the concrete sample when saturated and $m_{0}$ is the initial mass of the concrete sample after drying.

The porosity of concrete after $0,30,60$, and 90 freezethaw cycles was calculated to be $1.30 \%, 3.65 \%, 5.14 \%$, and $7.34 \%$, respectively, and a model of porosity $\phi_{n}$ varying with the number of freeze-thaw cycles $n$ was obtained, as shown in the following equation:

$$
\phi_{n}=\phi_{0} \eta e^{(\theta n)},
$$

where $\phi_{n}$ is the porosity of concrete after freeze-thawing; $\phi_{0}$ is the initial porosity of concrete before freeze-thawing; $\eta$ and $\theta$ are the material parameters, set at 1.61459 and 0.0143 , respectively; $n$ is the number of freeze-thaw cycles. The experimental values were compared with the calculated values, as shown in Figure 2.

\section{Corrosion Pattern of Chloride Ions in Concrete after Freeze-Thawing Cycles}

3.1. Test Methodology. The sample measured $100 \mathrm{~mm}$ $\times 100 \mathrm{~mm} \times 400 \mathrm{~mm}$; the materials and mix proportions are shown in Section 2.1, and the test procedure is shown in Figure 3. In the test, four groups (three samples in each group) were formed and treated with 0, 30, 60, and 90 freeze-thaw cycles, respectively (based on GBT50082-2009, shown in Figure 3(a)); subsequently, a $100 \mathrm{~mm} \times 400 \mathrm{~mm}$ rectangular surface of each sample was used as the corrosive surface, and the other surfaces were sealed with epoxy resin (shown in Figure 3(b)). The sealed samples were placed into an artificial marine climate simulation laboratory for salt spray corrosion test (shown in Figure 3(c)), and the humidity in the environmental chamber was controlled at $75 \%-80 \%$. The salt spray corrosion settings were as follows: salt spray “on" for $12 \mathrm{~h} / \mathrm{d}$ (hour/day) + salt spray “off” for $12 \mathrm{~h} / \mathrm{d}$ and $\mathrm{NaCl}$ mass concentration of $5 \%$ in the salt spray solution. When the corrosion time reached $30,110,190$, and $270 \mathrm{~d}$, the samples were removed, concrete powder was obtained by drilling to depths of $5,10,15,20,25,30,35$, and $40 \mathrm{~mm}$, respectively (shown in Figure $3(\mathrm{~d})$ ), and the chloride ion concentration values were measured using a DY-2501A chloride ion 
TABle 1: Composition of cement.

\begin{tabular}{lcccccccc}
\hline Materials & $\mathrm{SiO}_{2}(\%)$ & $\mathrm{Al}_{2} \mathrm{O}_{3}(\%)$ & $\mathrm{Fe}_{2} \mathrm{O}_{3}(\%)$ & $\mathrm{CaO}(\%)$ & $\mathrm{MgO}$ & $\mathrm{Na}_{2} \mathrm{O}(\%)$ & $\mathrm{K}_{2} \mathrm{O}(\%)$ & $\mathrm{SO}_{3}(\%)$ \\
\hline Cement & 21.6 & 4.13 & 4.57 & 64.47 & $1.06 \%$ & 0.11 & 0.56 & 1.74 \\
Fly ash & 55.6 & 25.8 & 6.90 & 8.70 & $1.80 \%$ & 0.30 & 0.10 & 0.60 \\
\hline
\end{tabular}
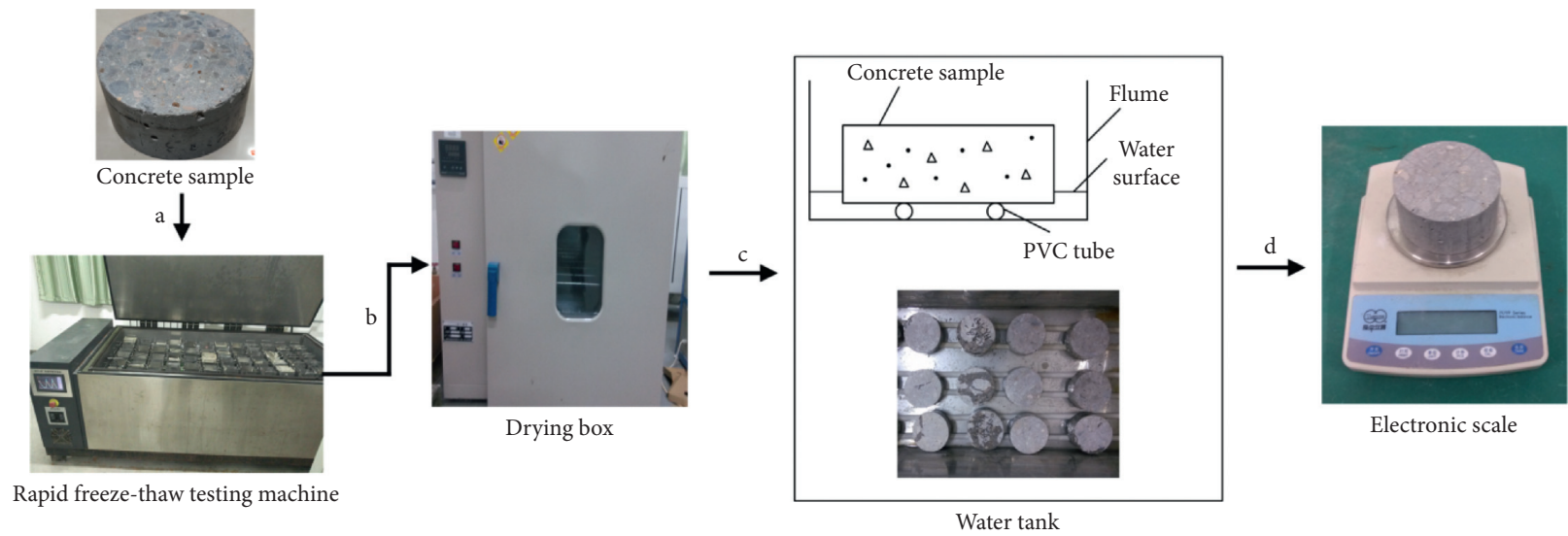

FIGURE 1: Schematic diagram of test procedure to determine water absorption performance.

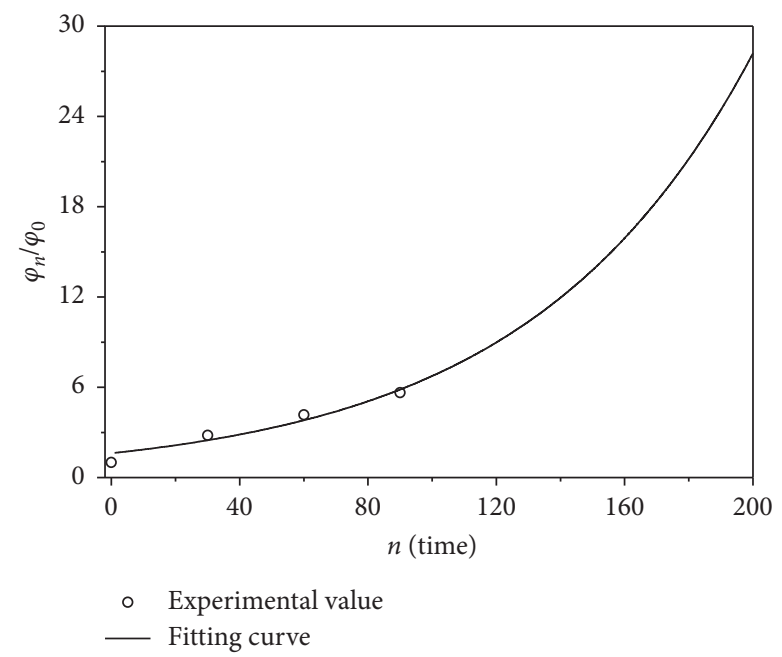

Figure 2: Porosity ratio $\left(\phi_{n} / \phi_{0}\right)$ varying with number of freeze-thaw cycles $(n)$.

concentration rapid tester (shown in Figure 3(e)). After each drilling of powder, the borehole was sealed with a sealant. Subsequently, the samples were placed back into the artificial marine climate simulation laboratory and continued to be corroded until the next testing time point (shown in Figure 3(f)), that is, until the end of $270 \mathrm{~d}$ of corrosion.

3.2. Chlorine Ion Concentration. Figures 4(a)-4(d) present the variation patterns of chloride ion concentration value $c$ with depth $l$ after $30,110,190$, and $270 \mathrm{~d}$ of chloride ion corrosion, respectively, the analysis of which shows that $c$ of the samples decreased with increasing $l$. In this experiment, the salt spray cycle corrosion mechanism was used, and the driving force for chloride ion intrusion into concrete was primarily provided by capillary action and concentration gradient. The chloride ion concentration gradient in the surface layer of concrete was relatively large, whereas the chloride ion concentration gradient in the deep layer was relatively small; hence, more chloride ions were accumulated in the surface layer of concrete. As the corrosion time progressed, the total chloride ion corrosion and corrosion depth increased. As the number of freeze-thaw cycles increased, the porosity of the concrete increased, the water and air permeability of the samples increased, chloride ions were more easily accessible and stored, and the corrosion concentration of chloride ions increased (at the same depth).

3.3. Chloride Ion Diffusion Coefficient. Fick's second law was used to describe the corrosion pattern of chloride ions in concrete, as shown in the following equation: 


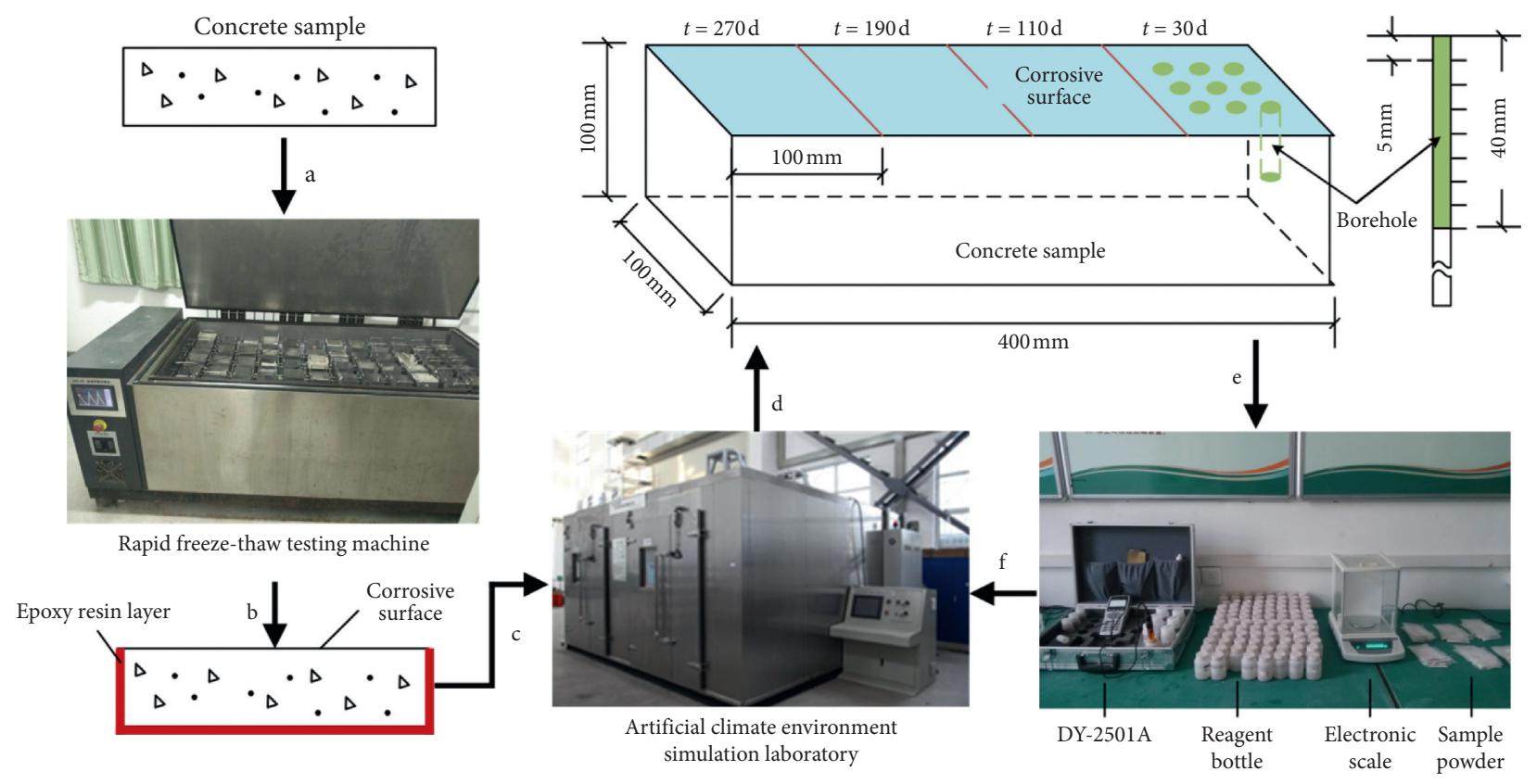

FIgURE 3: Flow chart of concrete chloride ion corrosion test.

$$
C(l, t)=C_{0}+\left(C_{s}-C_{0}\right)\left(1-\operatorname{erf} \frac{l}{2 \sqrt{D t}}\right) .
$$

$C_{0}$ is the initial chloride ion mass concentration at any section of concrete, $C_{\mathrm{s}}$ is the chloride ion mass concentration at the concrete surface at any moment $t, D$ is the chloride ion diffusion coefficient, $t$ is the time, $l$ is the distance from the concrete surface, and erf is the error function.

The inverse solution of equation (6) yields the equation for the chloride ion diffusion coefficient as follows:

$$
D=\frac{1}{t}\left\{\frac{l}{2 \operatorname{erf}^{-}\left[1-/ C(l, t)-C_{0} C_{s}-C_{0}\right]}\right\}^{2} .
$$

The mass concentration of chloride ions on the concrete surface $C_{s}$ is calculated as follows:

$$
c_{s}=\frac{\phi \rho_{c w} \omega}{\rho_{\mathrm{s}}+\phi \rho_{c w} \omega} \times \frac{35.5}{58.5} \times 100 \%,
$$

where $\phi$ is the porosity of concrete; $\rho_{\mathrm{w}}$ is the density of sodium chloride solution, that is, $1034 \mathrm{~kg} / \mathrm{m}^{3} ; \rho_{\mathrm{s}}$ is the density of concrete, that is, $2400 \mathrm{~kg} / \mathrm{m}^{3} ; \omega$ is the mass concentration of sodium chloride in the solution, that is, $5 \%$.

Based on equations (7) and (8), the chloride ion diffusion coefficient $D$ at $l=5 \mathrm{~mm}$ was calculated using MATLAB, and the results are shown in Table 2.

A mathematical model correlating the chloride ion diffusion coefficient $D_{n}$ with the number of freeze-thaw cycles $n$ was developed, as shown in the following equation:

$$
D_{n}=D_{0} \zeta e^{(\psi n)}
$$

where $D_{n}$ is the diffusion coefficient of concrete after freezethawing; $D_{0}$ is the diffusion coefficient of concrete before freeze-thawing; $\zeta$ and $\psi$ are the material parameters, which were set to 1.02151 and 0.00916 , respectively; $n$ is the number of freeze-thaw cycles. The experimental and calculated values were compared, as shown in Figure 5.

\section{Control Equations for Chloride Ion Transport in Concrete after Freeze- Thawing Cycles}

4.1. Basic Hypotheses. To derive the control equations for chloride ion transport in freeze-thaw corroded concrete, the following hypotheses are proposed: the liquid in concrete is an ideal liquid; the effect of temperature on liquid flow is not considered; and only a single solute (chloride ion) is considered. The transport of chloride ions in concrete can be classified into two main processes: solution flow and solute migration, in which the effects of freeze-thawing on both primarily affect the porosity and diffusion coefficient.

\subsection{Control Equation for Solution Flow}

\subsubsection{Mass Continuity Equation.}

$$
\frac{\partial(\rho \phi)}{\partial t}+\nabla \cdot(\rho \vec{v})=0
$$

where $\rho$ is the density of the solution, $\phi$ is the porosity, $t$ is the time, $\vec{v}=\left(v_{x}, v_{y}, v_{z}\right)^{\mathrm{T}}$, and $\nabla=((\partial / \partial x),(\partial / \partial y),(\partial / \partial z))$.

4.2.2. Porosity. Introducing the parameter $\xi$, pore deformation due to pore pressure changes can be characterized.

$$
\xi=\frac{1}{U_{w}} \frac{\mathrm{d} U_{w}}{\mathrm{~d} P}
$$

where $U_{w}$ denotes the pore volume.

The volume of each component phase of the porous medium is expressed as follows: 


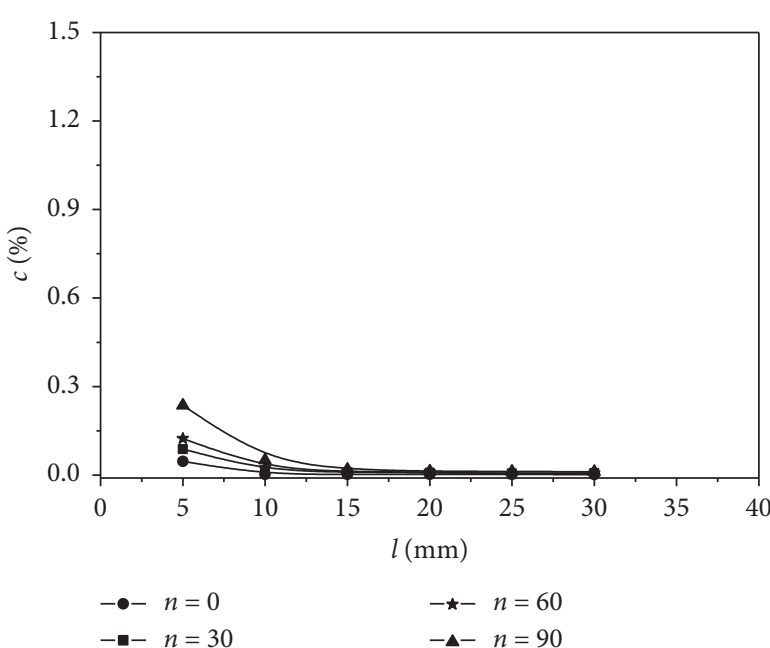

(a)

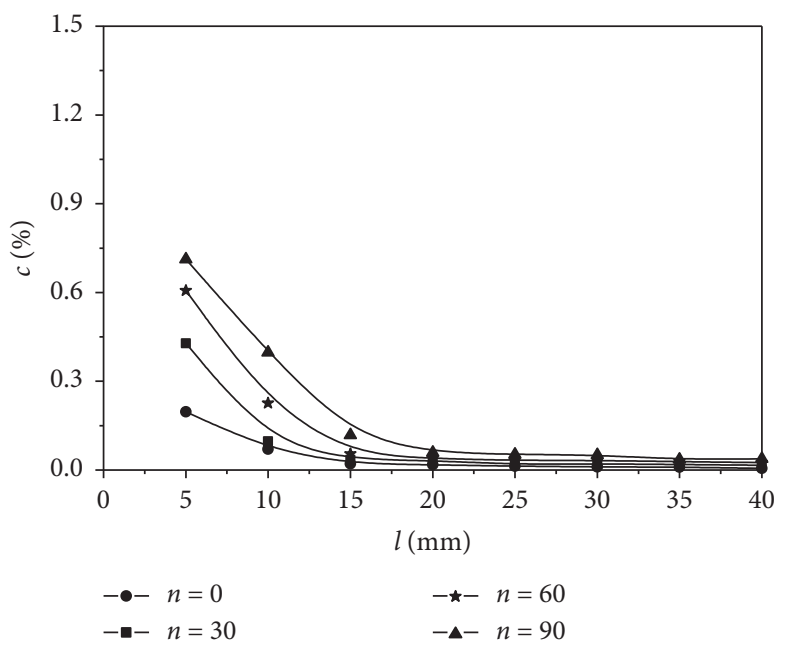

(c)

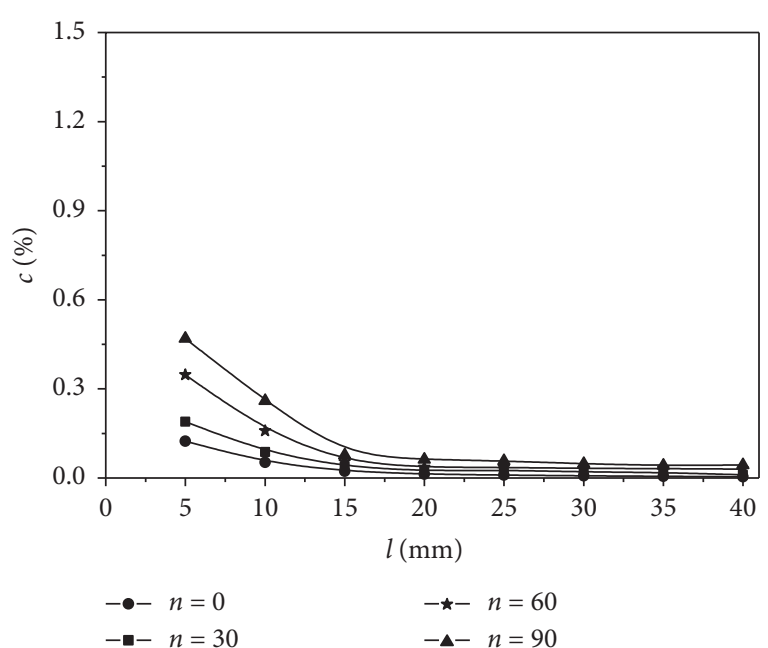

(b)

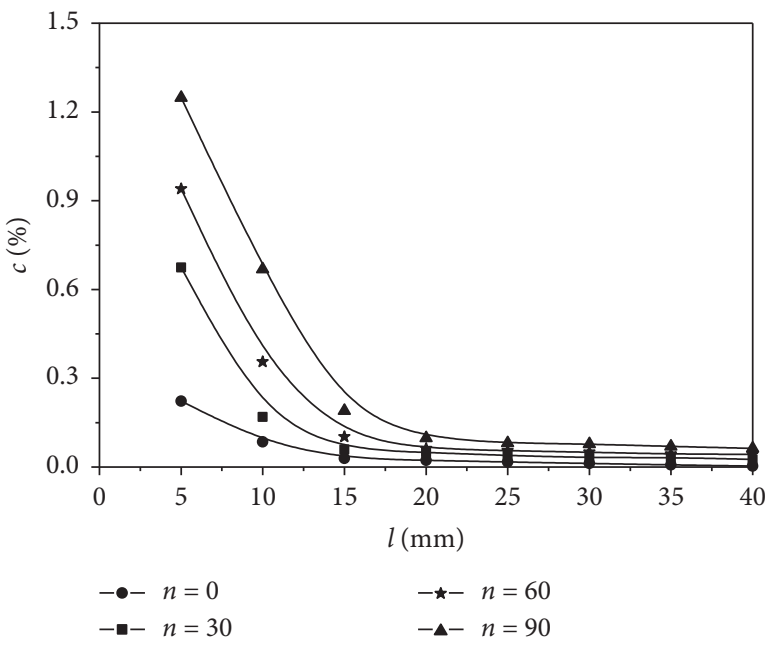

(d)

FIGURE 4: Variation pattern of concrete chloride ion concentration (c) with depth $(l)$ after freeze-thawing. (a) $t=30 \mathrm{~d}$. (b) $t=110 \mathrm{~d}$. (c) $t=190 \mathrm{~d}$. (d) $t=270 \mathrm{~d}$.

TABLe 2: Chloride ion diffusion coefficient $D$.

\begin{tabular}{lccccc}
\hline \multirow{2}{*}{ Number of freeze-thaw cycles $n /$ cycle } & \multicolumn{3}{c}{ Chloride ion diffusion coefficient $D /\left(\times 10^{-12} \mathrm{~m}^{2} / \mathrm{s}\right)$} & \multirow{2}{*}{ Mean value $\left(\times 10^{-12} \mathrm{~m}^{2} / \mathrm{s}\right)$} \\
\hline 0 & $t=30 \mathrm{~d}$ & $t=110 \mathrm{~d}$ & $t=190 \mathrm{~d}$ & $t=270 \mathrm{~d}$ & 0.3831 \\
30 & 0.8230 & 0.3162 & 0.2247 & 0.1683 & 0.5288 \\
40 & 1.0160 & 0.3812 & 0.3545 & 0.3636 & 0.6712 \\
90 & 1.1580 & 0.5320 & 0.4680 & 0.5267 & 0.8930 \\
\hline
\end{tabular}

$$
U=\frac{1}{\phi} U_{w}=U_{w}+U_{s}
$$

where $U$ is the total volume of the porous medium, $U_{s}$ is the volume of the solid skeleton of the porous medium, and $\phi$ is the porosity of the porous medium. Based on the above hypothesis that the solid particles do not undergo deformation, $U_{s}$ is a constant; that is, $\mathrm{d} U_{s}=0$.
Subsequently, equation (12) was used to obtain a model of porosity varying with pore pressure as follows:

$$
\phi=\frac{f(P)}{1+f(P)},
$$

where $f(P)=\left(\phi_{n 0} / 1+\phi_{n 0}\right) e^{\xi\left(P-P_{0}\right)}$ and $\phi_{n 0}$ is as shown in equation (5). 


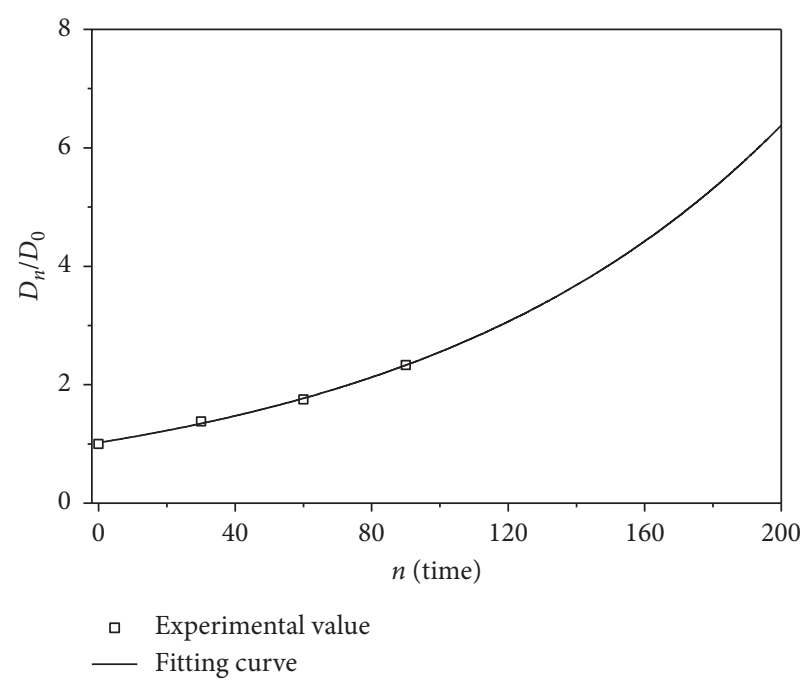

FIGURE 5: Variation curve of diffusion coefficient ratio $D_{n} /(\mathrm{D}) 0$ with respect to number of freeze-thaw cycles $(n)$.

4.2.3. Solution Flow Rate. It is assumed that the solution flow in the porous medium conforms to Darcy's law and that the effect of gravity is not considered.

$$
\vec{v}=-\frac{k}{\mu} \nabla P
$$

where $k$ is the permeability of the solution and $\mu$ is the dynamic viscosity of the solution.

The relationship between permeability and porosity is described using the Kotyakhov [38] model, as shown in the following equation:

$$
k=\frac{d^{2} \phi^{3}}{72(1-\phi)^{2}}
$$

where $d$ is the effective diameter of the porous medium particles. Based on equation (15), the following can be derived:

$$
\frac{k}{k_{0}}=\left(\frac{\phi}{\phi_{n}}\right)^{3}\left(\frac{1-\phi_{n}}{1-\phi}\right)^{2}
$$

where $k_{0}$ is the initial permeability of the solution. Because $1-\phi_{n} \approx 1$ and $1-\phi \approx 1$, equation (16) can be simplified as follows:

$$
\frac{k}{k_{0}}=\left(\frac{\phi}{\phi_{n 0}}\right)^{3}
$$

Therefore, based on equations (13) and (17), the solution velocity can be expressed as

$$
\vec{v}=-\frac{1}{\mu} \frac{k_{0}}{\phi_{n}^{3}}\left[\frac{f(P)}{1-f(P)}\right]^{3} \nabla P .
$$

4.2.4. Solution Density. As chloride ions are transported through concrete, the pore pressure and solution concentration change, resulting in changes in the solution density.
Hence, the density of solution $\rho$ is a function of the pore pressure $P$ and concentration $c$; that is,

$$
\rho=\rho(P, c) \text {. }
$$

Taking the derivative of both sides of equation (19) yields

$$
\mathrm{d} \rho=\frac{\partial \rho}{\partial P} \mathrm{~d} P+\frac{\partial \rho}{\partial c} \mathrm{~d} c
$$

To effectively characterize the density as a function of pore pressure and solution concentration, two parameters $\beta_{P}$ and $\beta_{c}$ were introduced, which are the pressure compression coefficient and concentration compression coefficient, respectively.

$$
\begin{aligned}
& \beta_{P}=\frac{1}{\rho} \frac{\mathrm{d} \rho}{\mathrm{d} P}, \\
& \beta_{c}=\frac{1}{\rho} \frac{\mathrm{d} \rho}{\mathrm{d} c} .
\end{aligned}
$$

Parameters $\beta_{P}$ and $\beta_{c}$ represent the ratio of the change in density due to a unit change in pressure to the initial density and the ratio of the change in density due to a unit change in concentration to the initial density, respectively.

Substituting equation (21) into equation (20) yields

$$
\mathrm{d} \rho=\rho\left(\beta_{P} \mathrm{~d} P+\beta_{c} \mathrm{~d} c\right)
$$

Integrating both sides of equation (22) yields

$$
\rho=\rho_{0} e^{\left[\beta_{P}\left(P-P_{0}\right)+\beta_{c}\left(c-c_{0}\right)\right]},
$$

where $\rho_{0}$ denotes the initial density, $P_{0}$ denotes the initial pore pressure, and $C_{0}$ denotes the initial concentration.

4.2.5. Control Equation for Solution Flow. Using equations (10), (13), (18), and (23), the flow control equation for chloride ion solution in concrete porous medium was obtained as follows:

$$
\left[\rho \phi \beta_{P}+\frac{\rho \xi \phi}{1+f(P)}\right] \frac{\partial P}{\partial t}+\rho \phi \beta_{c} \frac{\partial c}{\partial t}+\nabla \cdot\left(-\rho \frac{k}{u} \nabla P\right)=0 .
$$

4.3. Control Equation for Solute Migration. Chloride migration in concrete is governed by convection, molecular diffusion, mechanical dispersion, precipitation dissolution, complexation, and chemisorption. However, only the effects of convection, mechanical dispersion, and chemisorption on chloride migration were considered in this study.

4.3.1. Convective Effects. The convective migration of chloride ions is described by convective flux, as shown in the following equation:

$$
\vec{J}_{v}=\vec{u} c
$$

where $\vec{J}_{v}=\left(J_{v x}, J_{v y}, J_{v z}\right)^{\mathrm{T}}$ is the convective flow vector; $\vec{u}=\left(v_{x}, v_{y}, v_{z}\right)^{\mathrm{T}}$ is the solution flow velocity vector. 
4.3.2. Mechanical Dispersion. The mechanical dispersion migration flux of chloride ions is expressed by Fick's first law, as shown in the following equation:

$$
\vec{J}_{d}=-\phi\left[D_{d}\right] \forall c,
$$

where $\vec{J}_{d}=\left(J_{\mathrm{d} x}, J_{\mathrm{d} y}, J_{\mathrm{d} z}\right)^{\mathrm{T}}$ is the dispersion flux vector; $D_{d}=\left(D_{\mathrm{d} x}, D_{\mathrm{d} y}, D_{\mathrm{d} z}\right)^{\mathrm{T}}$ represents the mechanical dispersion coefficients in the $\mathrm{x}^{-}, \mathrm{y}^{-}$, and $\mathrm{z}$-directions, respectively. If we assume that the porous medium is isotropic, then $D_{d}=$ $\underline{D}_{\mathrm{d} x}=D_{\mathrm{d} y}=D_{\mathrm{d} z} \quad$ and $\quad J_{d}=J_{\mathrm{d} x}=J_{\mathrm{d} y}=J_{\mathrm{d} z} ;$ therefore, $\vec{J}_{d}=-\phi D_{d} \forall c$,

4.3.3. Chemisorption. The Langmuir adsorption theory was used to describe the chemisorption of chloride ions in concrete transport, as shown in the following equation:

$$
(1-\phi) \rho_{s} c_{k}=(1-\phi) \rho_{s} K_{L} \frac{C_{\max } c}{1+K_{L} c},
$$

where $\rho_{s}$ is the dry density of the skeleton material, $c_{\mathrm{k}}$ is the isothermal adsorption concentration, $K_{L}$ is the Langmuir isothermal nonlinear adsorption partition coefficient, and $C_{\max }$ is the maximum adsorption capacity.

\subsubsection{Control Equation for Solute Migration.}

$$
\frac{\partial(\phi c)}{\partial t}-\nabla \cdot(\phi[D] \cdot \nabla c)+\nabla \cdot(\vec{v} c)=0
$$

Equation (28) is the chloride ion convection-diffusion equation, where $[D]$ denotes the chloride ion diffusion coefficient tensor. It is assumed that the diffusion coefficient is uniform everywhere in the concrete; therefore, the diffusion coefficient can be expressed as shown in equation (9). Considering adsorption, the mass of chloride ions in the representative elementary volume is expressed as $\phi c-(1-$ b) $\rho_{s} K_{L}\left(C_{\max } c / 1+K_{L} c\right)$, and the convection-diffusion equation for chloride ions in concrete considering adsorption can be obtained by replacing $\phi c$ in equation (28) with the above equation; that is,

$$
\begin{aligned}
& {\left[c+\rho_{s} K_{L} \frac{C_{\max } c}{1+K_{L} c}\right] \frac{\partial \phi}{\partial t}+\left[\phi+(\phi-1) \rho_{s} K_{L} \frac{C_{\max }}{\left(1+K_{L} c\right)^{2}}\right] \frac{\partial c}{\partial t}} \\
& -\forall \cdot(\phi D \cdot \forall c)+\forall \cdot(\vec{v} c)=0 .
\end{aligned}
$$

Substituting equation (13) into equation (29) yields the convection-diffusion equation for chloride ions expressed in terms of pressure $P$ and concentration $c$, as in the following equation:

$$
\begin{gathered}
{\left[\xi\left(c+\rho_{s} K_{L} \frac{C_{\max } c}{1+K_{L} c}\right) \frac{\phi}{1+f(P)}\right] \frac{\partial P}{\partial t}} \\
+\left[\phi+(\phi-1) \rho_{s} K_{L} \frac{C_{\max }}{\left(1+K_{L} c\right)^{2}}\right] \frac{\partial c}{\partial t} \\
-\forall \cdot(\phi D \cdot \forall c)+\forall \cdot(\vec{v} c)=0 .
\end{gathered}
$$

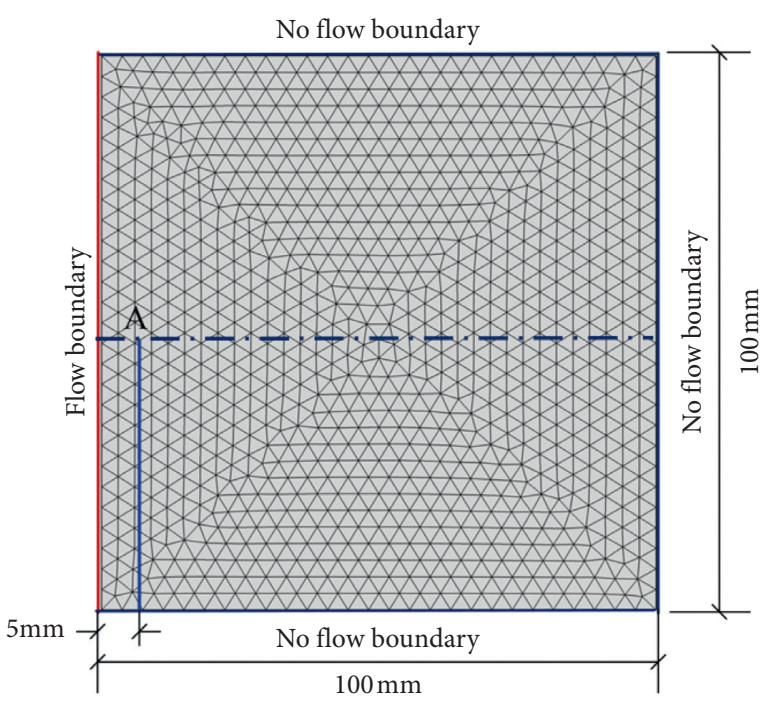

Figure 6: Numerical calculation model.

4.4. Control Equations for Chloride Ion Transport in Concrete. The control equations for chloride ion transport in concrete considering the freeze-thaw action comprise two parts, that is, the solution flow control equation and the solute migration control equation, as shown in $31 \mathrm{a}$ and $31 \mathrm{~b}$, respectively, which contain the effects of convection, diffusion, and chemisorption, reflecting the coupling between the seepage and solute migration fields, where $\phi$ and $D$ are shown in equations (13) and (9), respectively.

$$
\begin{aligned}
& {\left[\rho \phi \beta_{P}+\frac{\rho \xi \phi}{1+f(P)}\right] \frac{\partial P}{\partial t}+\rho \phi \beta_{c} \frac{\partial c}{\partial t}+\forall \cdot\left(-\rho \frac{k}{u} \forall P\right)=0} \\
& \quad+\left[\phi+(\phi-1) \rho_{s} K_{L} \frac{C_{\max }}{\left(1+K_{L} c\right)^{2}}\right] \frac{\partial c}{\partial t} \\
& \quad-\forall \cdot(\phi D \forall c)+\forall \cdot(\vec{v} c)=0 .
\end{aligned}
$$

The initial and boundary conditions are solved as follows

The solution flow and solute migration can be described using the Dirichlet and Neumann boundary conditions as follows:

$$
\begin{array}{ll}
p=\tilde{p}(t), \quad \vec{n} \cdot \frac{k}{\mu} \forall p=\widetilde{\mathrm{Q}}(t), \\
c=\tilde{c}(t), \quad \vec{n} \cdot D \forall c=F(t),
\end{array}
$$

where $\tilde{p}(t)$ and $\widetilde{Q}(t)$ are the fluid pressure and fluid flow velocity on the solution domain boundary $\partial \Omega ; \tilde{c}(t)$ and $F(t)$ are the chloride ion concentration and chloride ion diffusion flux on the solution domain boundary $\partial \Omega$, respectively. The initial conditions for fluid flow and chloride ion diffusion can be expressed as 


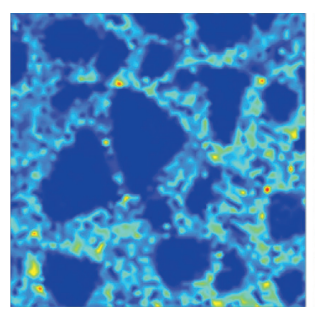

(a)

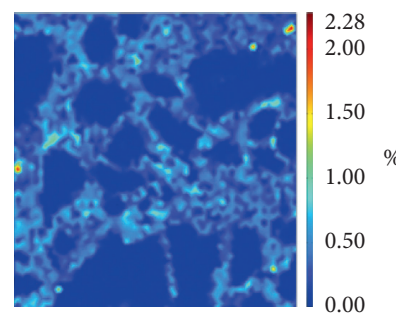

(b)

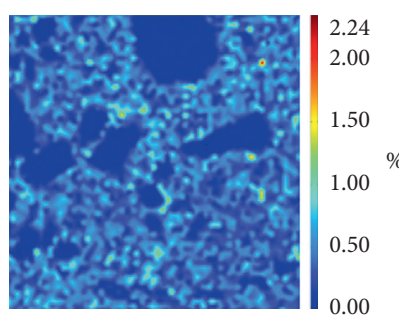

(c)

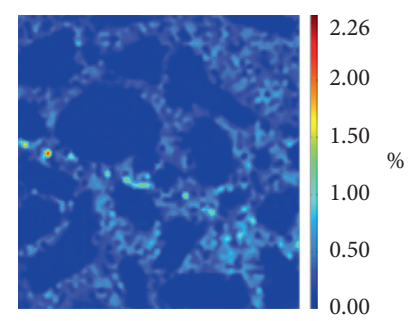

(d)

FiguRE 7: Cloud plots of initial porosity distribution of concrete after freeze-thawing. $(\mathrm{a})(n)=0$. (b) $(n)=3$. (c) $(n)=60$. $($ d) $(n)=90$.
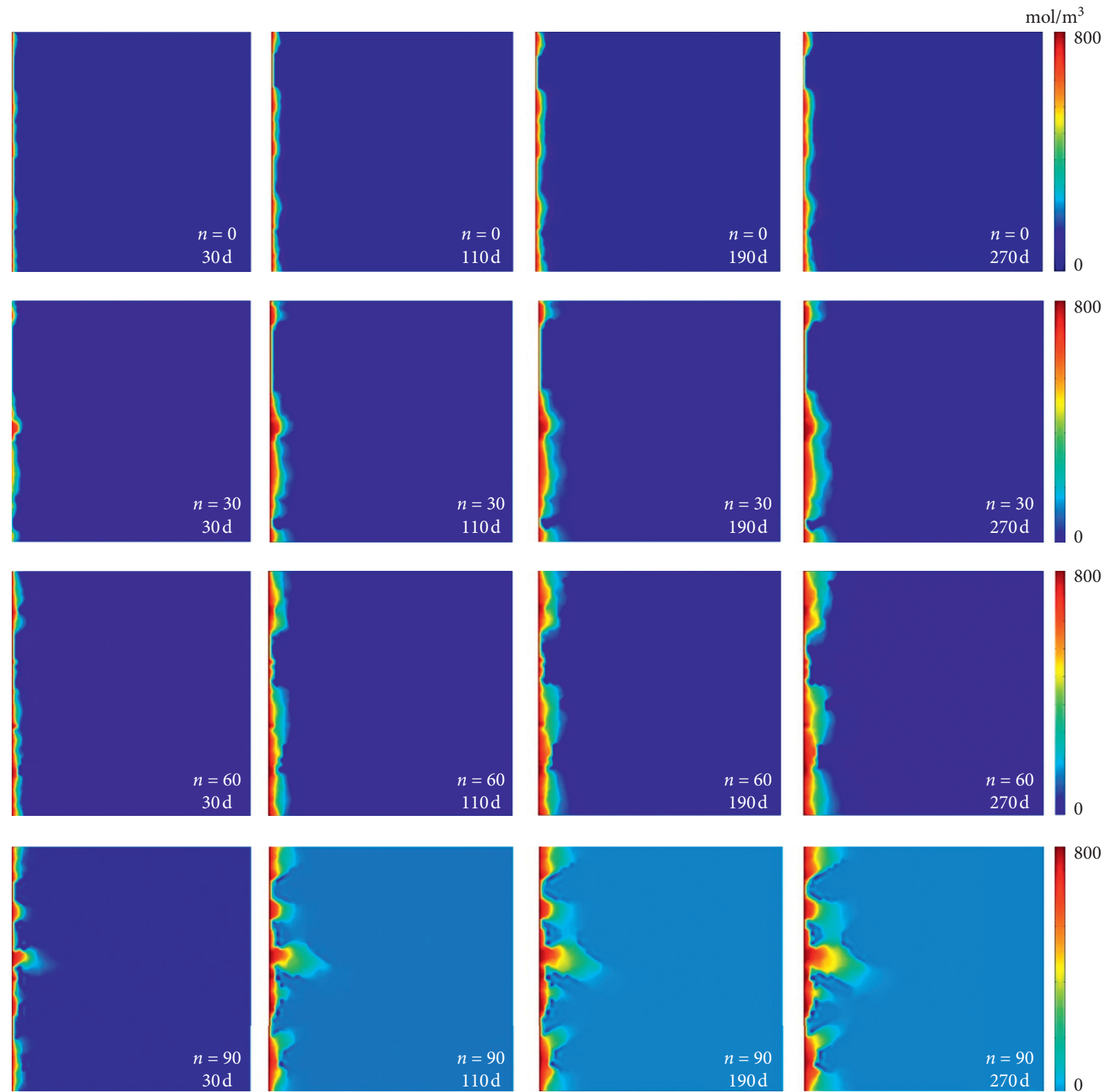

FIGURE 8: Cloud plots of chloride ion corrosion concentration distribution of concrete after freeze-thawing. 


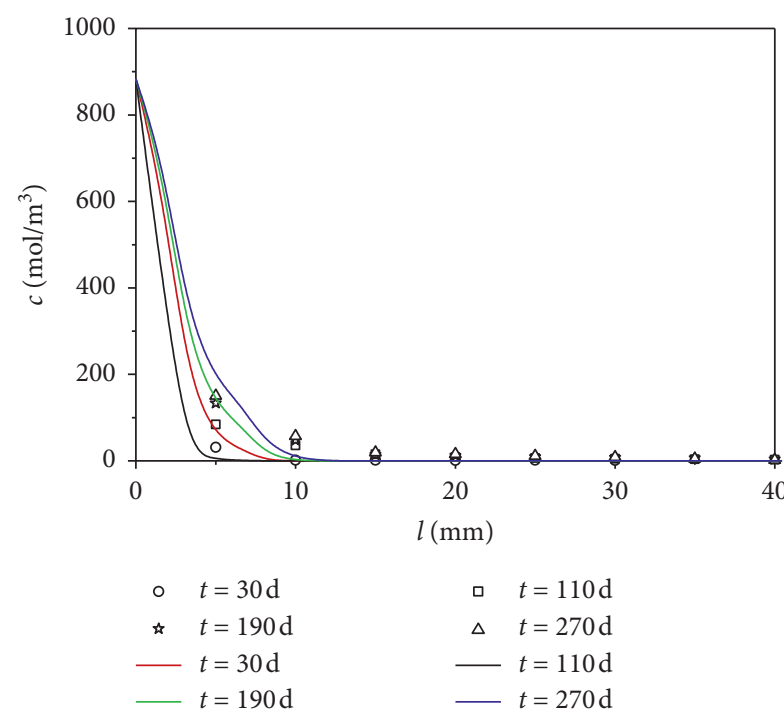

(a)

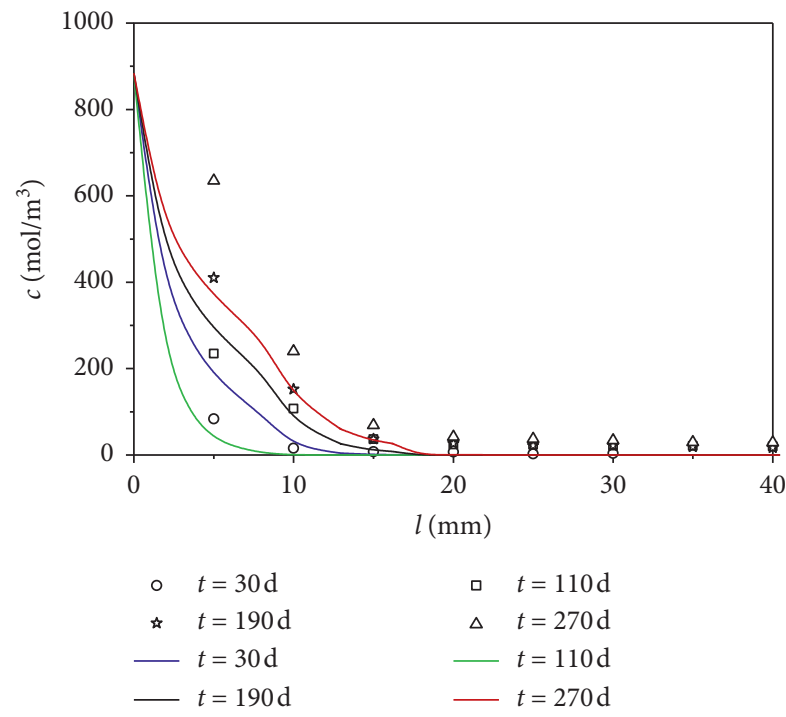

(c)

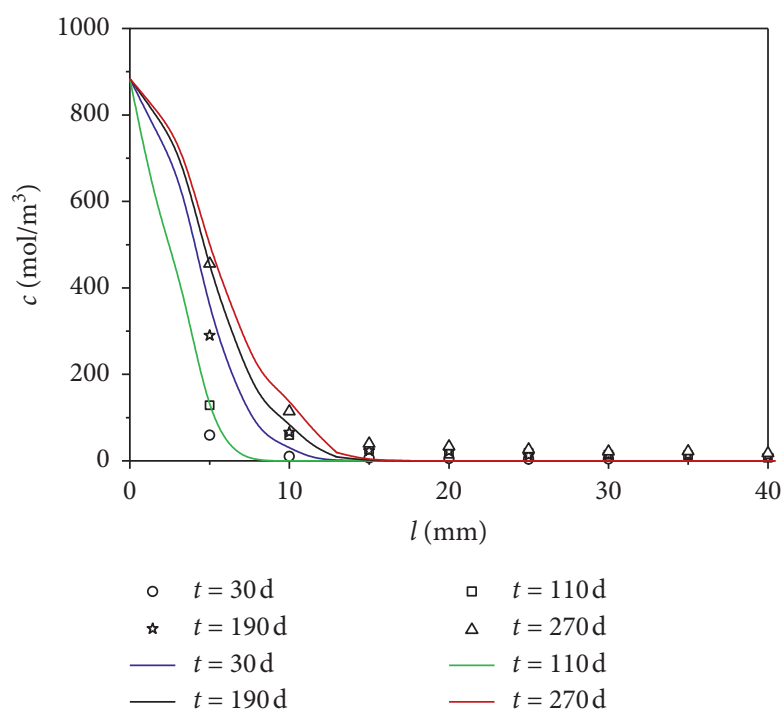

(b)

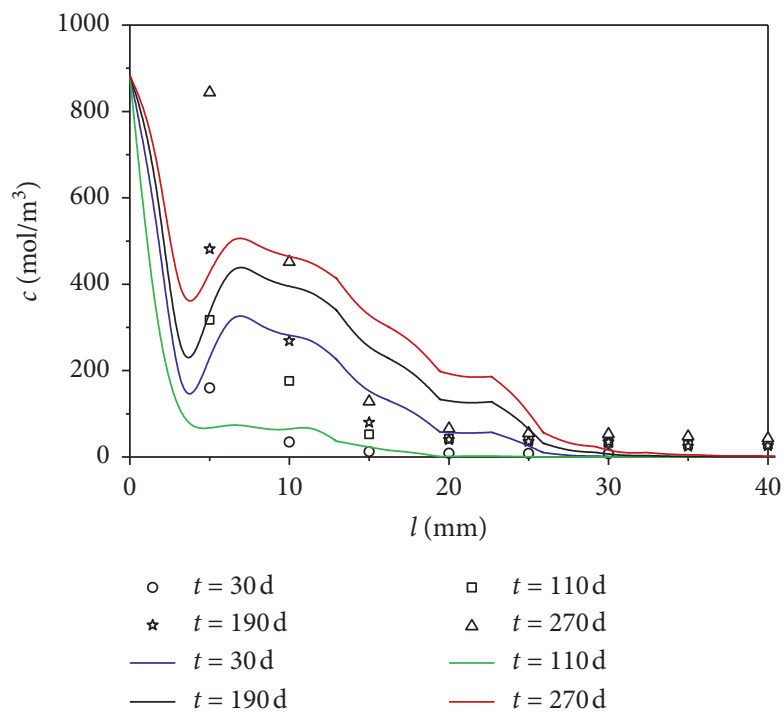

(d)

FIGURE 9: Numerically calculated curves of chloride ion concentration $c$ with depth $l$ compared with experimental values. (a) $n=0$. (b) $n=30$. (c) $n=60$. (d) $n=90$.

$$
P(0)=P_{0}, c(0)=c_{0}
$$

where $P_{0}$ and $c_{0}$ are the initial fluid pressure and chloride ion concentration distribution over the solution domain $\Omega$, respectively.

\section{Model Calculation and Validation}

\subsection{Calculation Scheme}

5.1.1. Numerical Model. Equations (31a) and (31b) were solved using COMSOL numerical calculation software to simulate the chloride ion corrosion in concrete after freezethawing. The numerical model measured $100 \mathrm{~mm} \times 100 \mathrm{~mm}$, the left boundary was set as the chloride ion corrosion boundary, and the other boundaries were no-flow boundaries. Point $\mathrm{A}$ is a point on the midline at a distance $5 \mathrm{~mm}$ from the left boundary, as shown in Figure 6. To study the effect of freeze-thaw concrete chloride ion corrosion, numerical simulation scenarios involving $0,30,60$, and 90 freeze-thaw cycles and 5\% chloride salt concentration were investigated. Because of the complex coupled model and boundary conditions, the solution was complex; therefore, the model parameters were partially simplified in the calculation; for example, the porosity $\phi$, solution density $\rho_{\mathrm{w}}$, chloride ion diffusion coefficient $D$, and other parameters were set as constants in the calculation.

5.1.2. Initial Pore Size Distribution. As shown in Figure 7, the processed numerical plots can accurately reflect the porefissure distribution in concrete, with smaller porosity 
corresponding to coarse aggregates and larger porosity corresponding to cracks. When the numbers of freeze-thaw cycles were 0,30 , and 60 , no cracks were observed in the concrete; therefore, the pores were relatively dispersed, and large pores dominated the mortar, as shown in Figures 7(a)$7(\mathrm{c})$; when the number of freeze-thaw cycles was 90, wider diagonal cracks appeared in the concrete, and the distribution of cracks can be observed well, as shown in Figure $7(d)$.

\subsection{Calculation Results and Analysis}

5.2.1. Concentration Distribution. Figure 8 shows the cloud plots of the chloride ion concentration distribution after 30 , 110,190 , and $270 \mathrm{~d}$ of chloride ion corrosion of concrete at a corrosive surface concentration of $854 \mathrm{~mol} / \mathrm{m}^{3}(5 \% \mathrm{NaCl}$ solution) when the numbers of freeze-thaw cycles $n$ were 0 , 30, 60, and 90, respectively. As shown, the chloride ions eroded nonuniformly from the surface to the interior; with the increase in the number of freeze-thaw cycles, chloride ion corrosion accelerated significantly, primarily due to the increased porosity of the concrete. Meanwhile, the chloride ion diffusion and permeation of the concrete after 90 freezethaw cycles were significantly faster than those of the concrete after 60 freeze-thaw cycles, primarily due to the increased porosity of the concrete and the generation of microcracks.

5.2.2. Chloride Ion Diffusion. Figure 9 shows the numerical simulation curves of chloride ion corrosion concentration $c$ in concrete with depth $l$ (horizontal model midline) after different numbers of freeze-thaw cycles compared with the experimental values, where the scatter points represent the experimental values, and the curves represent the simulated value. As shown, the simulated value of chloride ion concentration with depth was not a smooth decreasing curve owing to the introduction of the real concrete numerical model. Furthermore, the results reflected the effect of the coarse aggregates, as shown in Figure 9(d). The simulated curves deviated slightly from the experimental values, particularly in the smaller numbers of freeze-thaw cycles, as shown in Figures 9(a) and 9(b).

\section{Conclusion}

The porosities of C60 high-strength concrete after 0, 30, 60, and 90 freeze-thaw cycles determined using the water retention method were $1.30 \%, 3.65 \%, 5.14 \%$, and $7.34 \%$, respectively, and a mathematical model of porosity varying with the number of freeze-thaw cycles was developed.

The chloride ion diffusion patterns of C60 high-strength concrete after $0,30,60$, and 90 freeze-thaw cycles were obtained using an artificial environment simulation experimental system and the natural diffusion method, and the corresponding diffusion coefficients were calculated to be $0.3431 \times 10^{-12}$, $0.5288 \times 10^{-12}, 0.6712 \times 10^{-12}$, and $0.8930 \times 10^{-12} \mathrm{~m}^{2} / \mathrm{s}$, respectively. Furthermore, a mathematical model of diffusion coefficients varying with the number of freeze-thaw cycles was developed.
Transport control equations for chloride ions in concrete after freeze-thawing comprising a solution flow control equation and a solute migration control equation were developed, in which the effects of freeze-thawing, solution pressure, solution concentration, solution density, convective action, mechanical dispersion, and chemisorption on the transport of chloride ions in concrete were considered.

The chloride ion transport control equation was solved using a real concrete numerical calculation model in COMSOL numerical software to simulate the chloride ion corrosion process after different numbers of freeze-thaw cycles, and the simulated values of chloride ion concentration agreed well with the experimental values.

\section{Data Availability}

Some or all data, models, or code generated or used during the study are available from the corresponding author upon request.

\section{Conflicts of Interest}

The authors declare that there are no conflicts of interest regarding the publication of this paper.

\section{Acknowledgments}

The authors gratefully acknowledge the financial support for this study provided by the and 51974296), research project of “333 project" in Jiangsu Province of China (BRA2019236), Natural Science Research Project of Higher Education of Jiangsu (General Programs 16KJB560017, 17KJA560004, and 18KJB560019), Research Program of Xuzhou Institute of Technology (XKY2018131), Xuzhou Key Research and Development Program (KC18090 and KC20176), and Science and Technology Project of Xuzhou City (KC18241).

\section{References}

[1] W.-H. Yang, G.-X. Cui, G.-Q. Zhou, Y. Li, X.-D. Chen, and H.-L. Lv, "Fracture mechanism of shaft lining under special strata condition and the technique preventing the shaft from fracturing (Part One)," Journal of Chnia University of Mining and Technology, vol. 25, no. 4, pp. 1-5, 1996.

[2] L.-F. Dai and J. Tan, "Development and application of the special mine shaft sinking technology to coal mines in China," Coal Engineering (China), vol. 45, no. 12, pp. 9-12, 2013.

[3] Q. Yu, J. Ma, H. Shimada, and T. Sasaoka, "Influence of coal extraction operation on shaft lining stability in eastern Chinese coal mines," Geotechnical and Geological Engineering, vol. 32, no. 4, pp. 821-827, 2014.

[4] Y. Chen, H. Lin, X. Ding, and S. Xie, "Scale effect of shear mechanical properties of non-penetrating horizontal rocklike joints," Environmental Earth Sciences, vol. 80, p. 192, 2021.

[5] C.-Y. Zhang, C.-Z. Pu, R.-H. Cao, T.-T. Jiang, and G. Huang, "The stability and roof-support optimization of roadways passing through unfavorable geological bodies using advanced detection and monitoring methods, among others," Bulletin of Engineering Geology and the Environment, vol. 78, no. 7, pp. 5087-5099, 2019.

[6] C.-Y. Zhang, Y.-X. Wang, H. Ruan, B. Ke, and H. Lin, "The strain characteristics and corresponding model of rock 
materials under uniaxial cyclic load/unload compression and their deformation and fatigue damage analysis," Archive of Applied Mechanics, 2021.

[7] C.-Y. Zhang, Y.-X. Wang, and T.-T. Jiang, "The propagation mechanism of an oblique straight crack in A rock sample and the effect of osmotic pressure under in-plane biaxial compression," Arabian Journal of Geosciences, vol. 13, no. 15, p. 736, 2020.

[8] Z.-M. He, D. Xiang, Y.-X. Liu, Q.-F. Gao, and H.-B. Bian, "Deformation behavior of coarse-grained soil as an embankment filler under cyclic loading," Advances in Civil Engineering, vol. 2020, Article ID 4629105, 13 pages, 2020.

[9] Z.-M. He, Z.-F. Li, X.-H. Liu, and H.-B. Bian, "Improved method for determining active earth pressure considering arching effect and actual slip surface," Journal of Central South University, vol. 27, no. 7, pp. 2032-2042, 2020.

[10] R.-H. Cao, H. Lin, Q. Lin, and J. Meng, "Failure mechanism of non-persistent jointed rock-like specimens under uniaxial loading: laboratory testing," International Journal of Rock Mechanics and Mining Sciences, vol. 132, Article ID 104341, 2020.

[11] R.-H. Cao, R.-B. Yao, T. Hu, C.-S. Wang, K.-H. Li, and J.-J. Meng, "Failure and mechanical behavior of transversely isotropic rock under compression-shear tests: laboratory testing and numerical simulation," Engineering Fracture Mechanics, vol. 241, Article ID 107389, 2021.

[12] R.-H. Cao, C.-S. Wang, R.-B. Yao et al., "Effects of Cyclic Freeze-thaw Treatments on the Fracture Characteristics of Sandstone under Different Fracture Modes: Laboratory Testing," Theoretical and Applied Fracture Mechanics, vol. 109, Article ID 102738, 2020.

[13] X.-S. Chen, "Time-space design theory for deep ice wall of short cylinder," Chinese Journal of Geotechnical Engineering, vol. 20, no. 5, pp. 13-16, 1998.

[14] R.-J. Chen, G.-D. Cheng, S.-X. Li, X.-M. Guo, and L.-N. Zhu, "Development and prospect of research on application of artificial ground freezing," Chinese Journal of Geotechnical Engineering, vol. 22, no. 1, pp. 40-44, 2000.

[15] T. Wang, F.-T. Yue, Y.-D. Jiang, and T.-Q. Zheng, "Research and practice on forced thaw Technology applied to frozen wall of mine shaft," Journal of China Coal Society, vol. 35, no. 6, pp. 918-922, 2010.

[16] Z.-S. Yao, H. Cheng, and J.-J. Yang, "The experimental study on high strength reinforced concrete shift lining in deep alluvium," Journal of China Coal Society, vol. 29, no. 4, pp. 167-171, 2004.

[17] Z.-S. Yao, "An experimental study on steel fiber reinforced high strength concrete shaft lining in deep alluvium," Chinese Journal of Rock Mechanics and Engineering, vol. 24, no. 7, pp. 1253-1258, 2005.

[18] Z.-S. Yao, H. Cheng, and C.-X. Rong, "Experimental study on composite shaft lining of inner steel plate cylinder and high strength reinforced concrete in deep frozen shaft," Chinese Journal of Rock Mechanics and Engineering, vol. 27, no. 1, pp. 154-160, 2008.

[19] Z.-S. Yao, H. Cheng, and C.-X. Rong, "Shaft structural design and optimization of deep freezing bedrock shaft in west area," Journal of China Coal Society, vol. 35, no. 5, pp. 760-764, 2010.

[20] B. Li, H. Yin, X. Mao et al., "Macroscopic and microscopic fracture features of concrete used in coal mine under chlorine salt erosion," International Journal of Mining Science and Technology, vol. 26, no. 3, pp. 455-459, 2016.

[21] C. Zhang, W.-H. Yang, J.-H. Liu, Z.-C. Wei, and H.-P. Li, "Construction Technology and monitoring analysis on concrete strain of a new single-layer shaft lining in deep aqueous bed rock during freeze sinking," Journal of China Coal Society, vol. 37, no. 2, pp. 192-199, 2012.
[22] Y.-F. Gao, K.-M. Liu, H. Xie, K.-P. Gao, B.-H. Chen, and F.-Y. Zhang, "Steel tube concrete support applied to dynamic pressure roadway in kilometers deep mine," Coal Science and Technology, vol. 43, no. 8, pp. 7-11, 2015.

[23] B. Li, L.-Y. Zhang, Y. Li, H.-G. Yin, and R.-X. Liu, "Stepwise loading-unloading creep testing of fly ash concrete and its constitutive model," Thermal Science, vol. 23, no. 3 Part A, pp. 1539-1545, 2019.

[24] W. Lu, B. Jiang, Q. Wang, H.-B. Sun, Y. B. Huang, and P. Zhang, "Mechanical characteristics and parameter influencing mechanism of square steel confined concrete arch components in deep soft rock roadway," Journal of Mining \& Safety Engineering, vol. 37, no. 3, pp. 473-480, 2020.

[25] J.-L. Liu, L.-W. Chen, and J.-L. Wang, "Design method for shaft wall strength of coal mine considering influence of temperature stress," Chinese Journal of Rock Mechanics and Engineering, vol. 30, no. 8, pp. 1577-1563, 2011.

[26] J.-L. Liu, L.-W. Chen, and J.-L. Wang, "Characteristic analysis of temperature stresses of shaft wall," Rock and Soil Mechanics, vol. 32, no. 8, pp. 2389-2390, 2011.

[27] L.-W. Jing and H.-L. Li, "The affection caused by variance of temperature to the strength of shaft wall in shafts constructed by freezing method," Journal of China Coal Society, vol. 25, no. 1, pp. 40-44, 2000.

[28] L.-W. Jing and H. Zhang, "Fracture analysis and prevention measures of fraction initiation in the melting stage of frozen shaft wall," Engineering Mechanics, vol. 20, no. 1, pp. 122-126, 2003.

[29] L.-W. Jing, Q.-C. Gao, H.-D. Xu, and R.-S. Yang, "Thermal stress analysis of shaft in melting stage of frozen wall," Rock and Soil Mechanics, vol. 25, no. 9, pp. 1357-1362, 2004.

[30] L.-W. Jing, Q.-C. Gao, F. Liu, and X.-C. Niu, "Rupture Mechanism and Rupture Forecast of The Shaft Wall in Mine," Engineering Mechanics, vol. 23, no. 3, pp. 156-161, 2006.

[31] Y.-S. Wang, Z.-J. Yang, Y.-F. Yang, and C.-G. Zhang, "Mechanics model and ultimate horizontal bear capacity of outer shaft lining during freezing sinking," Journal of Mining and Safety Engineering, vol. 26, no. 3, pp. 359-366, 2009.

[32] Y.-S. Wang, J.-P. Cheng, L.-B. Xue, Y.-L. Ren, Z.-J. Yang, and J.-H. Li, "In-situ measurements and analysis of frost-heave pressure inside and outside the ice wall during freeze sinking," Journal of China University of Mining and Technology, vol. 38, no. 3, pp. 303-308, 2009.

[33] Y. Yi, D.-J. Zhu, S.-C. Guo, Z.-H. Zhang, and C.-J. Shi, “A review on the deterioration and approaches to enhance the durability of concrete in the marine environment," Cement and Concrete Composites, vol. 113, Article ID 103695, 2020.

[34] S. Yin, J. Liu, S.-Y. Zhou, and G.-P. Li, “"Experimental investigation on the freeze-thaw durability of concrete under compressive load and with joints," Construction and Building Materials, vol. 229, Article ID 116893, 2019.

[35] M. Shafikhani and S. E. Chidiac, "Quantification of concrete chloride diffusion coefficient-a critical review," Cement and Concrete Composites, vol. 99, pp. 225-250, 2019.

[36] S. Yin, L. Jing, M. Yin, and B. Wang, "Mechanical properties of textile reinforced concrete under chloride wet-dry and freeze-thaw cycle environments," Cement and Concrete Composites, vol. 96, pp. 118-127, 2019.

[37] R.-X. Liu, H.-G. Yin, L.-Y. Zhang, B. Li, and X.-B. Mao, "Model for the patterns of salt-spray-induced chloride corrosion in concretes under coupling action of cyclic loading and salt spray corrosion," Processes, vol. 7, no. 84, 2019.

[38] G. V. Chilingar, "Relationship between porosity, permeability, and grain-size distribution of sands and sandstones," Developments in Sedimentology, vol. 1, pp. 71-75, 1964. 